\title{
Exercise heart rate recovery in obesity
}

\section{(DLada Bradić1*, -Martina Lovrić Benčić, (D) Juraj Jug'2, (D) Martina Matovinovićc', -Kristina Gašparović', DRea Levicki ${ }^{3}$}

'University of Zagreb School of Medicine, University Hospital Centre Zagreb, Zagreb, Croatia

${ }^{2}$ University of Zagreb School of Medicine, Zagreb, Croatia ${ }^{3}$ Požega General County Hospital, Požega, Croatia

\section{RECEIVED:}

August 2, 2019

ACCEPTED:

September 16, 2019

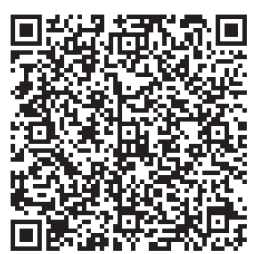

KEYWORDS: obesity, heart rate recovery, autonomic dysfunction

CITATION: Cardiol Croat. 2019;14(9-10):233. | https://doi.org/10.15836/ccar2019.233

*ADDRESS FOR CORRESPONDENCE: Lada Bradić, Klinički bolnički centar Zagreb, Kišpatićeva 12, HR-10000 Zagreb, Croatia. / Phone: +385-91-5794-115 / E-mail: lada.bradic@gmail.com

ORCID: Lada Bradić, https://orcid.org/0000-0001-8296-699x • Martina Lovrić Benčić, https://orcid.org/0000-0001-8446-6120 Juraj Jug, https://orcid.org/0000-0002-3189-1518 • Martina Matovinović, https://orcid.org/0000-0002-6325-7394 Kristina Gašparović, https://orcid.org/0000-0002-1191-4831 • Rea Levicki, https://orcid.org/0000-0003-3687-1310

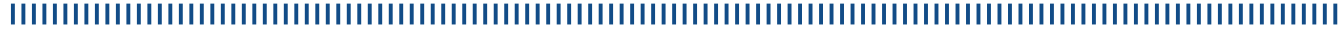

Introduction: Obesity is an epidemic that carries significant cardiovascular (CV) burden. Autonomic dysfunction, characterized by reduced vagal tone and sympathetic overactivity, has been found in diabetes, hypertension, heart failure, metabolic syndrome, and other conditions. Heart rate decrease after exercise, or heart rate recovery (HRR) reflects cardiac autonomic activity. Decreased HRR is a powerful predictor of CV disease, CV and all-cause mortality. ${ }^{1-3}$

Patients and Methods: A total of 54 obese patients (24\% male, 76\% female), age 22-66 (mean 47 year) BMI 29.4-53.3 (mean $40.3 \mathrm{~kg} / \mathrm{m}^{2}$ ), were enrolled in a multidisciplinary weight management program. Treadmill testing was done initially and after 6-month follow-up. Standard Bruce protocol was used to assess exercise capacity and passive HRR ( $15 \mathrm{sec}, 3$ and 6 min into the recovery period, as per institutional protocol). Control group was composed of age- and sex-matched non-obese subjects.

Results: Obese subjects had significantly slower HRR (HRR0:15 6.1 vs. 9.1 BPM, HRR3:00 57.8 vs. 66.6 BPM, HRR6:00 63.3 vs. 72.7 BPM; p=0.0216, 0.0006, 0.0004, respectively). Exercise capacity was also significantly lower in comparison to control (6.7 vs. 8.6 METs; $p=0.000001)$, with reduced exercise time (6.0 vs. $8.2 \mathrm{~min}$; $\mathrm{p}=0.000001)$. Sixteen subjects that reached 6 -month follow-up lost $5.4 \mathrm{~kg}$ on average. Exercise capacity increased mildly (6.4 to 7.1 METs; NS), as well as total exercise time (5.6 to 6.6 min; NS). Discussion and Conclusion: Physiological HRR kinetics follows exponential decay function. Rapid first phase, mediated by vagal reactivation is followed by a gradual HRR decline, dominated by sympathetic withdrawal. We found significantly slower HRR over different time-points in the obese, which reflects autonomic imbalance. Functional aerobic capacity was also significantly reduced. Somewhat improved functional capacity and dynamics of HRR after weight loss did not reach statistical power. To conclude, we found evidence of significantly impaired cardiac autonomic function in obese subjects, together with reduced functional capacity. As the study is ongoing, we hope to demonstrate sustained effect of exercise and diet on autonomic function. Potential benefit on mortality and CV risk reduction should encourage patients and health care providers to manage obesity more vigorously.

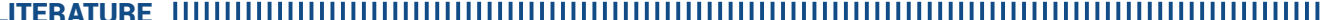

1. Peçanha T, Silva-Júnior ND, Forjaz CL. Heart rate recovery: autonomic determinants, methods of assessment and association with mortality and cardiovascular diseases. Clin Physiol Funct Imaging. 2014 Sep;34(5):327-39. https://doi.org/10.1111/cpf.12102

2. Pierpont $\mathrm{GL}$, Adabag S, Yannopoulos D. Pathophysiology of exercise heart rate recovery: a comprehensive analysis. Ann Noninvasive Electrocardiol. 2013 Mar;:18(2):107-17. https://doi.org/10.1111/anec.1206

3. Sydó N, Sydó T, Gonzalez Carta KA, Hussain N, Farooq S, Murphy JG, et al. Prognostic Performance of Heart Rate Recovery on an Exercise Test in a Primary Prevention Population. J Am Heart Assoc. 2018 Mar 26;7(7). pii: e008143. https://doi.org/10.1161/JAHA.117.008143 